\title{
Influence of Main Road Alignment on Handling Stability of Runaway Vehicle on Truck Escape Ramp
}

\author{
Pinpin Qin ${ }^{1}$, Yaqi Han ${ }^{1}$, Heyu Hu${ }^{2}$, Guangyi QIN ${ }^{1}$ and Jie WANG ${ }^{1}$ \\ ${ }^{1}$ College of Mechanical Engineering, Guangxi University, Nanning, Guangxi, China \\ ${ }^{2}$ RWS Group, Mechanics Department, No. 76 of West Yanta Road, Xi'an, Shaanxi, China
}

\begin{abstract}
Main road alignment exerts an influence on handling stability of a runaway vehicle to a certain extent, as rational designs of the main road alignment play an important role in ensuring that a runaway vehicle enters a truck escape ramp safely. Firstly, forces acting on the runaway vehicle on the main road are analysed, and the minimum curve radius value and the corresponding approach angle that can prevent lateral rollover and lateral slip are discussed. Secondly, a simulation software, TruckSim, is used to establish a simulation model of the runaway vehicle and the road, wherein the minimum curve radius value and the corresponding approach angle that can prevent lateral rollover and slip are taken as input parameters of the road model. Finally, the established simulation model of the vehicle and road is used to simulate the influence that three types of main road alignment, i.e. a straight line, a right curve and a left curve, respectively have on the handling stability of the runaway vehicle entering a truck escape ramp.
\end{abstract}

\section{Introduction}

A truck escape ramp is usually arranged on one side of a long, steep downhill grade section of a road, and is used to guide a runaway vehicle or trailer with a brake failure from the main road into the truck escape ramp. In the United States, studies of truck escape ramps are mainly conducted in the 1980s, during which a series of research results were achieved to guide the design and application of truck escape ramps [1-4]. Both the research and application of truck escape ramps in China lag far behind those at abroad; moreover, engineering norms or design guidelines have not been introduced yet. There are still some deficiencies in the design of current truck escape ramps, which have led to a variety of accidents of runaway vehicles on the truck escape ramps, such as a vehicle rushing out of a truck escape ramp, a rollover of a vehicle on an arrester bed, goods squeezing into a cab, backward sliding of a vehicle on an arrester bed, and a vehicle crashing into a guardrail or the other vehicles on a truck escape ramp [5-6].

Research triggered by these accidents is directed to the safety of truck escape ramps, and studies on truck escape ramps in China mainly focus on the design of the location, the cross-section width, the approach angle and approach roads, the deflection angle, the vertical curve radius at the entrance, etc. of truck escape ramps [7-13]. Few studies have investigated the influence of design parameters of the truck escape ramp on the safety performance of a runaway vehicle; however, the safety performance of a truck escape ramp should further include the handling stability of a runaway vehicle when entering the truck escape ramp. This research expects to find out the main vehicle dynamics indicators that reflect the handling stability of a vehicle, so as to provide the basis for the design of main road alignment.

\section{Research method}

\subsection{Force analysis of runaway vehicle on main road}

The vehicle running on a horizontal curve produces a centrifugal force, which is directed away from the centre of the circle, and the magnitude of which is directly proportional to the square of the travelling speed and inversely proportional to the radius of the horizontal curve. The calculation formula is as follows:

$$
F=\frac{G v^{2}}{g R}
$$

wherein $\mathrm{F}$ is the centrifugal force; $\mathrm{R}$ is the radius of a horizontal curve; $\mathrm{v}$ is the travelling speed of a runaway vehicle; and $\mathrm{G}$ is the gravity.

The lateral force analysis of a runaway vehicle at a horizontal curve is shown in Fig. 1, in which, $\mathrm{F}$ is the centrifugal force, $\mathrm{G}$ is the gravity of the runaway vehicle, and ih is the superelevation slope. The tread of the runaway vehicle is $b$, and the height of the centre of gravity thereof from the road surface is $h$. 


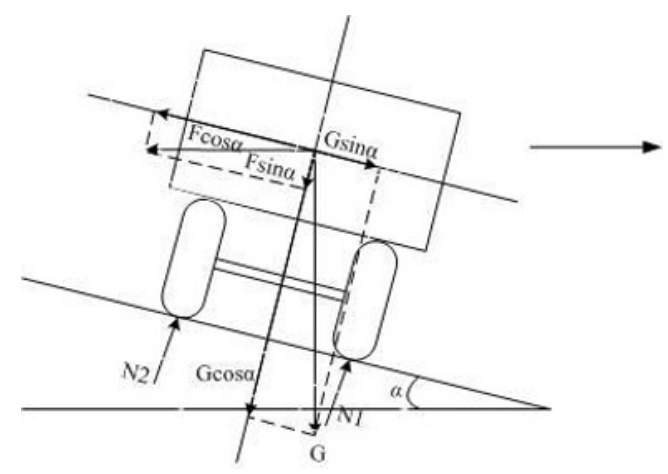

Figure. 1 Lateral force analysis of runaway vehicle at horizontal curve

The centrifugal force $F$ and the runaway vehicle gravity $\mathrm{G}$ are decomposed into a horizontal force parallel to the road surface and a vertical force perpendicular to the road surface, and thus the lateral stability balance formula is as follows:

$$
F \cdot \cos \alpha-\mathrm{G} \cdot \sin \alpha=F_{s}
$$

As lateral inclination angle of the road surface is generally small, then

$$
\frac{G v^{2}}{g R}=G \cdot \varphi+G \cdot i_{h}=G\left(\varphi+i_{h}\right)
$$

wherein $\varphi$ is the lateral adhesion coefficient of the road surface.

When the vehicle speed is greater than a certain value, the runaway vehicle may slip outward and cause an accident. In order to prevent the runaway vehicle from sideslipping, the travelling speed must satisfy the following equation:

$$
v \leq \sqrt{\left(\varphi+i_{h}\right) g R}
$$

When runaway vehicles are driving on a horizontal curve with superelevation, there is also a risk of lateral rollover around the point of contact between the outer wheel and the road. In order to prevent runaway vehicle from overturning, the roll moment must be less than or equal to the stabilizing moment, i.e.

$$
(F \cdot \cos \alpha-G \cdot \sin \alpha) \cdot h \leq(F \cdot \sin \alpha+G \cdot \cos \alpha) \cdot \frac{b}{2}
$$

Substituting $\sin \alpha=\tan \alpha=i_{h}, \cos \alpha \approx 1$ into the above equation, since $F i_{h}$ is much smaller than $G$ and thus negligible, the following equation is obtained:

$$
\frac{G v^{2}}{g R} \leq G\left(i_{h}+\frac{b}{2 h}\right)
$$

Therefore, in order to prevent the lateral rollover of a runaway vehicle on a horizontal curve, the travelling speed thereof must satisfy the following equation:

$$
v \leq \sqrt{\left(i_{h}+\frac{b}{2 h}\right) g R}
$$

According to the steering characteristics of the runaway vehicle driving from the main road into the truck escape ramp, the trajectory can be approximately considered as a horizontal curve, as shown in Fig. $2^{[5]}$. According to geometric principles, the relationship between the approach angle $\theta$ of the truck escape ramps, the length $\mathrm{L}$ of the transition section and the turning radius $\mathrm{R}$ are as follows:

$$
\begin{gathered}
\theta=\cos ^{-1}\left(\frac{R-3.75 / 2}{R+B / 2}\right) \\
L=\left(R+\frac{B}{2}\right) \sin \theta
\end{gathered}
$$

wherein $B$ is the width of the arrester bed.

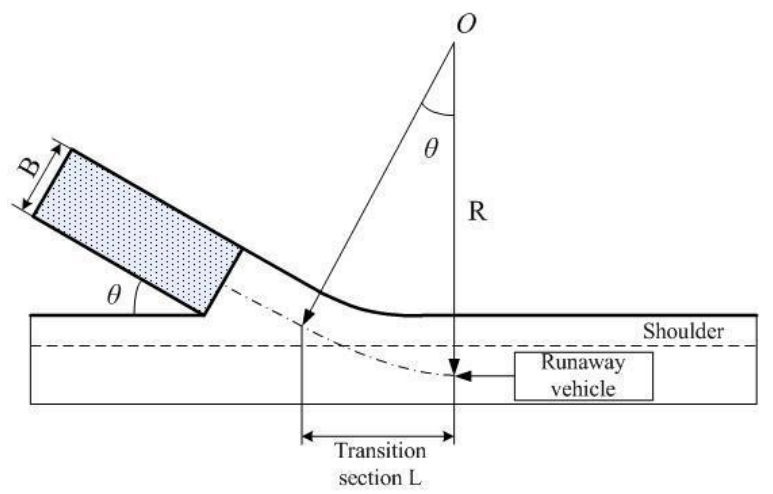

Figure. 2 Travelling trajectory of runaway vehicle entering truck escape ramp

\subsection{Determination of minimum curve radius and approach angle}

The maximum speeds that a runaway vehicle can reach in the case of different long, steep downhill grade sections are not the same. For the sake of safety, it is assumed that the speeds of the runaway vehicle entering truck escape ramps are $90 \mathrm{~km} / \mathrm{h}, 108 \mathrm{~km} / \mathrm{h}, 120 \mathrm{~km} / \mathrm{h}$ and $125 \mathrm{~km} / \mathrm{h}$ respectively. It is assumed that the tread of the runaway vehicle is $1.815 \mathrm{~m}$, and the height of the centre of gravity thereof from the road surface is $1.25 \mathrm{~m}$. The width of the road surface is assumed as $3.75 \mathrm{~m}$. The coefficient of road adhesion is related to the condition of a road surface, tyres and vehicle speeds, and considering the most unfavourable situation, the lateral adhesion coefficient of the road surface is assumed as 0.24 . Since it is provided that the superelevation is no more than $10 \%$, superelevation rates of $2 \%, 4 \%, 6 \%$ and $8 \%$ are selected herein, based on which the minimum curve radius that can prevent a lateral rollover and lateral slip of a runaway vehicle under different combinations are calculated, as shown in Tab. 1.

Table. 1 Minimum curve radius that can prevent lateral rollover and lateral slip of vehicle

\begin{tabular}{|c|c|c|c|c|}
\hline Approach speed $(\mathrm{km} / \mathrm{h})$ & 90 & 108 & 120 & 125 \\
\hline \multirow{2}{*}{ Superelevation 8\% } & 79 & 114 & 141 & 153 \\
\cline { 2 - 5 } & 199 & 287 & 354 & 384 \\
\hline \multirow{2}{*}{ superelevation 6\% } & 81 & 117 & 144 & 157 \\
\cline { 2 - 5 } & 212 & 306 & 377 & 410 \\
\hline \multirow{2}{*}{ superelevation 4\% } & 83 & 120 & 148 & 161 \\
\cline { 2 - 5 } & 227 & 328 & 404 & 439 \\
\hline \multirow{2}{*}{ superelevation 2\% } & 85 & 123 & 152 & 165 \\
\cline { 2 - 5 } & 245 & 353 & 436 & 473 \\
\hline
\end{tabular}


According to equations (1)-(9), the minimum trajectory circle radius, i.e. $\mathrm{R}$, of the runaway vehicle entering the truck escape ramp is calculated, so as to determine the maximum approach angle of the truck escape ramp corresponding to different situations (wherein the incoming speed is $120 \mathrm{~km} / \mathrm{h}$ ) for calculation, and the results are shown in Tab. 2.

Table. 2 Approach angle of truck escape ramp (incoming speed: $120 \mathrm{~km} / \mathrm{h}$ )

\begin{tabular}{|c|c|c|}
\hline $\begin{array}{c}\text { Approach } \\
\text { angle/ }\left(^{\circ}\right)\end{array}$ & $\begin{array}{c}\text { Length of } \\
\text { transition } /(\mathrm{m})\end{array}$ & $\begin{array}{c}\text { Turning radius } \\
/(\mathrm{m})\end{array}$ \\
\hline 2.5 & 254 & 5768 \\
\hline 5 & 249 & 2915 \\
\hline 7.5 & 105 & 797 \\
\hline 10 & 75 & 426 \\
\hline 12.5 & 65 & 296 \\
\hline 15 & 55 & 207 \\
\hline
\end{tabular}

\section{Simulation experiment}

\subsection{Establishment of vehicle simulation model}

In order to make the test result closer to the actual situation, a Balong Heavy Truck of Dongfeng Liuzhou Motor, which is a 385-horsepower 6x4 dump truck having a large market share, is selected as the simulation vehicle. Users can set and adjust vehicle model parameters in TruckSim as needed.

\subsection{Establishment of road simulation model}

With reference to the relevant parameters in Tab.3. TruckSim is used to establish truck escape ramp models. Users can set and adjust the road parameters of the truck escape ramp, such as the length of a transition section, the approach road, horizontal alignment, deflection and grade, in TruckSim according to requirements.

Table. 3 Truck escape ramp parameter

\begin{tabular}{|c|c|}
\hline Width of approach/(m) & $3.7 \sim 5.5$ \\
\hline Width of shoulder/(m) & 0.75 \\
\hline Width of arrester bed $/(\mathrm{m})$ & $7.5 \sim 12$ \\
\hline
\end{tabular}

\subsection{Experimental design}

In this paper, the main road alignment is divided into three different types of alignment, i.e. a straight line, a right horizontal curve and a left horizontal curve, to simulate the process of a runaway vehicle entering a truck escape ramp. On the premise that the runaway vehicle meets the stability requirement of the minimum radius when entering the truck escape ramp from a main road, the approach angle is set as $5^{\circ}, 10^{\circ}$ and $15^{\circ}$, and the vehicle speed is $120 \mathrm{~km} / \mathrm{h}$. In order to study the stability of the vehicle during the incoming process, four vehicle dynamics responses, i.e. the lateral acceleration, the yaw rate, the tyre slip angle and the roll angle, are taken as the indicators for evaluation.

\section{Analysis of simulation result}

\subsection{Main road alignment being straight line}

In the case of the main road alignment being a straight line, the simulation results of the lateral acceleration, yaw rate, tyre slip angle and roll angle of the runaway vehicle entering the truck escape ramp are shown in Fig. 3.

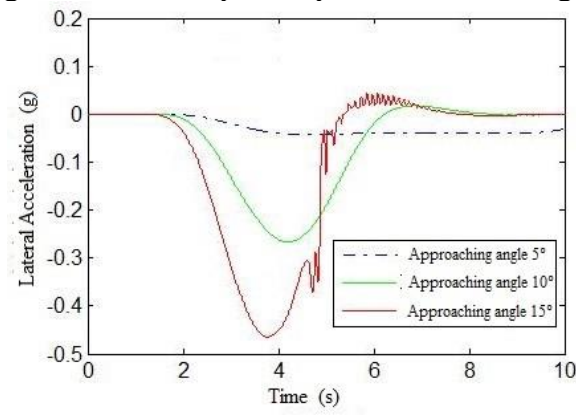

(a) Vehicle lateral acceleration over time

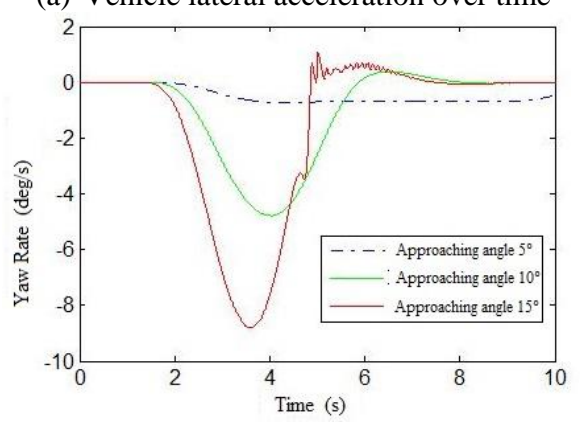

(b) Vehicle yaw rate over time

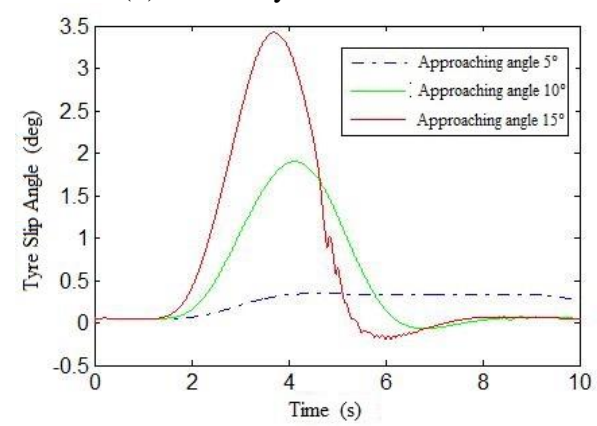

(c) Tyre slip angle over time

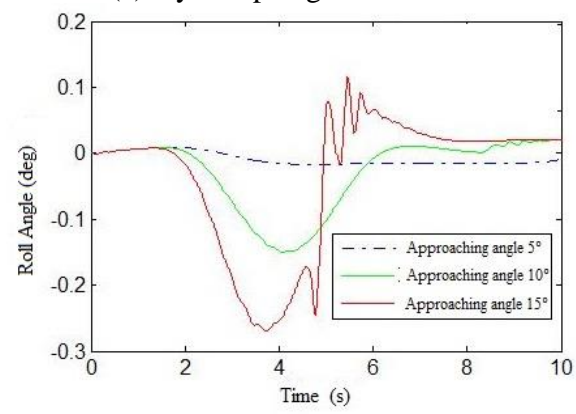

(d) Vehicle roll angle over time

Figure. 3 Simulation result for straight line main road 
From the simulation results in Fig. 3(a), it can be seen that when the runaway vehicle enters the truck escape ramp from the straight main road, the lateral acceleration increases gradually as the runaway vehicle approaches the entrance of the truck escape ramp. Moreover, as the approach angle increases, the increase of lateral acceleration is more obvious. When the approach angle is $15^{\circ}$, the lateral acceleration of the runaway vehicle is of a large magnitude that is close to $0.5 \mathrm{~g}$, in which case the vehicle is in a dangerous situation and the vehicle is close to a rollover state. After the runaway vehicle enters the approach road of the truck escape ramp, the lateral acceleration of the vehicle gradually decreases. However, the vehicle in the whole incoming process is not stable, and a rollover may occur.

From the simulation results in Fig. 3(b), it can be seen that the yaw rate of the vehicle increases with the increase of the approach angle of truck escape ramps, and the regularity is obvious. The yaw rate of the vehicle does not change much when the approach angle is $5^{\circ}$, whereas the yaw rate thereof reaches $9 \mathrm{deg} / \mathrm{s}$ when the approach angle is $15^{\circ}$ and the vehicle enters the approach road.

From the simulation results in Fig. 3(c), it can be seen that the tyre slip angle is constantly increasing in the process of the runaway vehicle entering the truck escape ramp. When the runaway vehicle enters the entrance of the truck escape ramp with an approach angle of $15^{\circ}$, the tyre slip angle is large. Although the tyre slip angle does not exceed the determination threshold, it becomes more difficult for the driver to handle the vehicle.

From the simulation results in Fig. 3(d), it can be seen that the roll angle of the vehicle increases gradually as the runaway vehicle enters the truck escape ramp from the straight main road. Moreover, as the approach angle increases, the increase of the roll angle of the vehicle is more obvious. When the approach angle is $15^{\circ}$, the roll angle of the vehicle is close to $0.3 \mathrm{deg}$, in which case the vehicle is in a dangerous situation, since the vehicle body tilts to one side, and the driver is unable to control the posture of the vehicle body. After the runaway vehicle enters the approach road of the truck escape ramp, the roll angle of the vehicle gradually decreases. However, the vehicle in the whole incoming process is not stable, and a rollover may occur.

\subsection{Main road alignment being right curve}

In the case of the main road alignment being a right curve, the simulation results of the lateral acceleration, yaw rate, tyre slip angle and roll angle of the runaway vehicle entering the truck escape ramp are shown in Fig. 4.

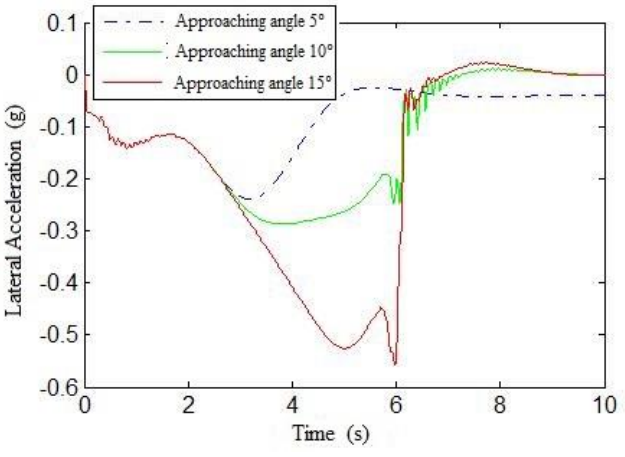

(a) Vehicle lateral acceleration over time

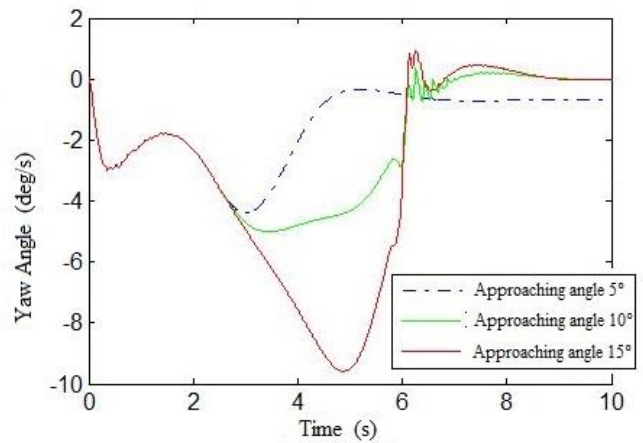

(b) Vehicle yaw rate over time

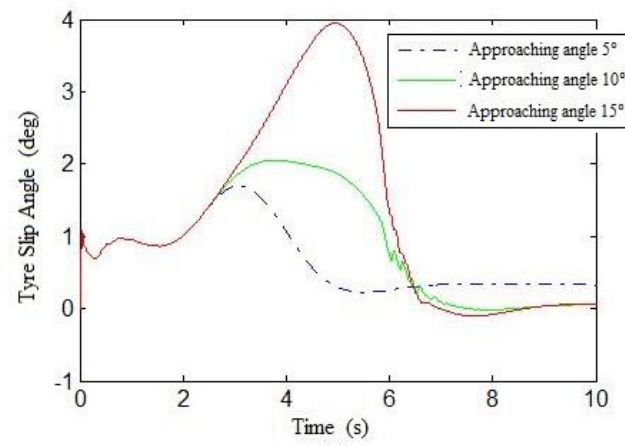

(c) Tyre slip angle over time

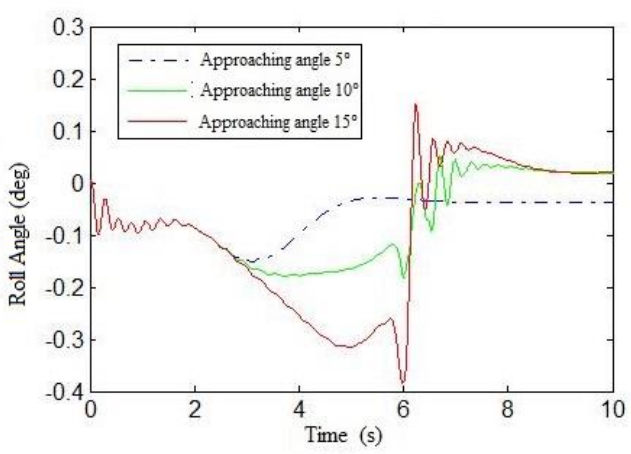

(d) Vehicle roll angle over time

Figure. 4 Simulation result for right curve main road

From the simulation results in Fig. 4(a), the lateral acceleration increases gradually as the runaway vehicle approaches the entrance of the truck escape ramp, and the lateral acceleration is already of a large magnitude when the runaway vehicle reaches the entrance, where the vehicle is in a dangerous situation, and although the instability state of the vehicle does not occur, it is already very difficult for the driver to control the vehicle at this 
time. After the runaway vehicle enters the approach road of the truck escape ramp, the lateral acceleration of the vehicle gradually decreases. However, the vehicle in the whole incoming process is not stable, and a rollover is very likely to occur.

From the simulation results of Fig. 4(b), it can be seen that as the runaway vehicle enters the truck escape ramp, the yaw rate increases to a certain extent, then decreases gradually, and finally becomes stable.

From the simulation results in Fig. 4(c), it can be seen that the tyre slip angle is constantly increasing in the process of the runaway vehicle entering the truck escape ramp. When the runaway vehicle reaches the entrance of the truck escape ramp, the value of the tyre slip angle is large, which indicates that the driver has some difficulties in handling the vehicle at this time; however, the tyre slip angle does not exceed the determination threshold of $5^{\circ}$, the state of instability of the vehicle does not occur.

From the simulation results in Fig. 4(d), it can be seen that the roll angle of the vehicle increases gradually as the runaway vehicle enters the truck escape ramp from the straight main road. Moreover, as the approach angle increases, the increase of the roll angle of the vehicle is more obvious. When the approach angle is $15^{\circ}$, the roll angle of the vehicle is close to $0.4 \mathrm{deg}$, in which case the vehicle body obviously tilts to one side, the vehicle is in a dangerous situation, and the posture of the vehicle is out of control. After the runaway vehicle enters the approach road of the truck escape ramp, the roll angle of the vehicle gradually decreases. However, the vehicle in the whole incoming process is very unstable, and a rollover of the vehicle is most likely to occur in this case.

The analysis of the above simulation data shows that the runaway vehicle did not lose its stability when the runaway vehicle enters the truck escape ramp with the limit radius of the right curve at the speed of $120 \mathrm{~km} / \mathrm{h}$. Although the tyre slip angle and vehicle roll angle are relatively large when the vehicle reaches the entrance of the truck escape ramp, the tyre slip angle and vehicle roll angle do not exceed the determination threshold, and experienced drivers can generally control the vehicle. To some extent, the simulation experiment data support the fact that the value of the limit radius of the right turn curve is rational in theory.

\subsection{Main road alignment being left curve}

When the main road alignment is a left curve and the truck escape ramp is arranged in a tangent direction of the main road, the runaway vehicle drives into the truck escape ramp along a straight line, in which case the lateral acceleration, yaw rate, tyre slip angle and roll angle are very small, so that the handling stability of runaway vehicle is equivalent to that of the vehicle travelling along a straight line. It is known from the engineering experience that it is the safest to arrange the truck escape ramp in the tangent direction of a left curve.

\section{Conclusion}

The influence of main road alignment on the handling stability of a runaway vehicle is well reflected by the four vehicle dynamics responses, i.e. the lateral acceleration, the yaw rate, the tyre slip angle and the roll angle. When the minimum curve radius that can prevent a lateral rollover and lateral slip of a runaway vehicle is used as an input parameter of the road simulation model, the runaway vehicle in the entering process is extremely unstable in the case of the main road alignment being a right curve and the approach angle being $15^{\circ}$. Therefore, the minimum curve radius that theoretically does not cause a lateral rollover and lateral slip is underestimated in the actual travelling process. The response indicators of vehicle dynamics can also be used as the basis to determine whether the design of main road alignment is rational and feasible. Only if the safety and stability of runaway vehicles are ensured during the process of entering a truck escape ramp from a main road, can the truck escape ramp achieve its design purpose.

\section{References}

1. Eck, Ronald W. Development of warrants for the use and location of truck escape ramps. Volume $\mathbf{I}$ - final report [R].1980.

2. Eck, Ronald W. Development of warrants for the use and location of truck escape ramps. Volume II appendices [R].1980.

3. J.C. Wambold, L.A. Rivera-Ortiz and M.C. Wang and Wambold, James C. A field and laboratory study to establish truck escape ramp design methodology. Volume I, Executive summary [R].1988.

4. J.C. Wambold, L.A. Rivera-Ortiz and M.C. Wang and Wambold, James C. A field and laboratory study to establish truck escape ramp design methodology. Volume II, Final report [R].1988.

5. Zhang Jianjun, Zhang Gaoqiang. Analysis of incident in emergency escape ramps and legal risk aversion [J].ICTE,2013:706-715.

6. MA Zhuanglin, ZHANG Honglu, TAN Xiaowei. Temporal-spatial distribution characteristics of traffic accidents on freeway continuous downgrade segment[J]. China Safety Science Journal, 2014, (05): 85-91.

7. GUO Xin, XU Yang, LIU Mingwen, et al. Research on safety of truck escape ramps on continuous long steep downgrades[J]. China Safety Science Journal, 2014, 24(4):152-158.

8. HUANG Xiucheng, TIAN Xidong, SUN Zhiyong. Safety evaluation of truck escape ramps of Guangdong Jiangxi Expressway[J]. Highway, 2005(12):102-104.

9. NIU Jianfeng, WANG Junhua, DONG Xianyuan. Research on the angle to truck escape ramps of mountain roads $[\mathrm{J}]$. Journal of Chongqing Jiaotong University (Natural Science), 2010, (04):604-608.

10. PAN Binghong. Minimum radius of vertical curve at the entrance of truck escape ramps [J]. Highway, 2011, (06):35-38.

11. SONG Cancan, GUO Zhongyin, LIN Lin. Departure angle and approach length of truck escape $\operatorname{ramp}[\mathrm{J}]$. 
Journal of Tongji University, 2016, 4(44)587-592.

12. SONG Cancan, GUO Zhongying. Width of cross section on truck escape ramp based on driving simulation[J]. Journal of Tongji University (Natural Science), 2017, (01):46-52.
13. LI Hai, LIU Xiaoyong, SHI Hongxing. Preliminary analysis on the method of installation for drift angle on truck escape ramp[J]. Highway Engineering. 2013(6):84-86. 